\title{
Regional Computing Culture and Personalities
}

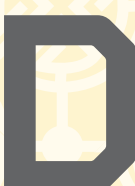

ESPITE THE GREAT Firewall, China's 772 million Internet users are adept at using smartphones for social media, live streaming, ordering home delivery, booking taxis, and sharing bicycles. Despite this massive market and bountiful opportunities for computing careers, Chinese corporations face great obstacles in attracting high-tech talent.

In April 2018, Chinese tech giants Baidu, Alibaba, and Tencent were called out for sexism in their recruitment campaigns. ${ }^{a}$ These corporate computing leaders were criticized

a https://www.hrw.org/reapply/gender-discriminationjob-advertisements-china port/2018/04/23/only-men-need-

by the members of Human Rights Watch for advertising male-only jobs and for portraying women as "goddesses" to entice young male programmers to apply. The companies apologized and promised remedial action, but the flawed practice reflects a serious challenge in China: The world's most populous country does not have enough technologists to drive its lofty tech ambitions.

The average computer science doctoral graduate ${ }^{\mathrm{b}}$ in China earns 121,000 yuan $(\$ 19,000)$ a year, and those with AI skills can command 300,000 to 500,000 yuan. "There continues to be a

\section{b 7http://www.chinadaily.com. cn/a/201806/02/WS5b11977e- a31001b82571dc75.html}

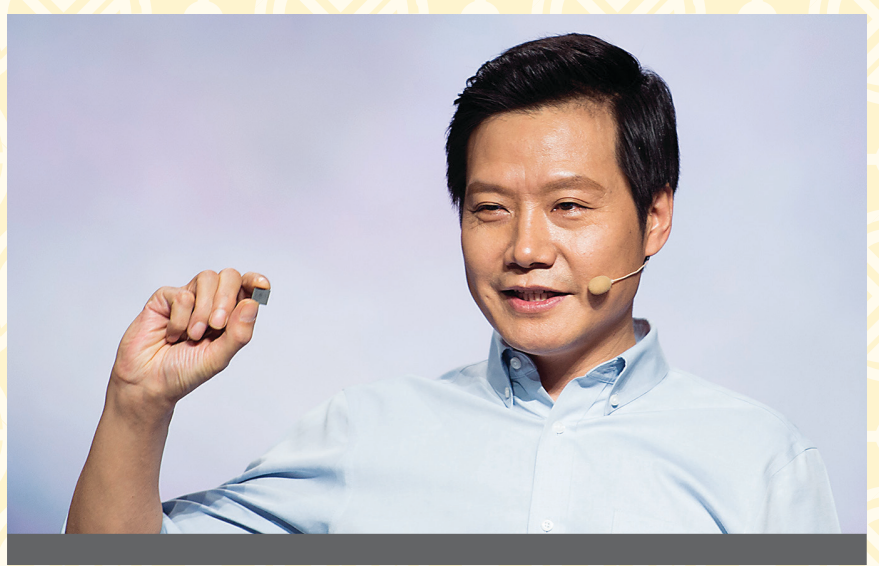

Lei Jun, CEO and co-founder of Xiaomi, is a role model for young technologists.

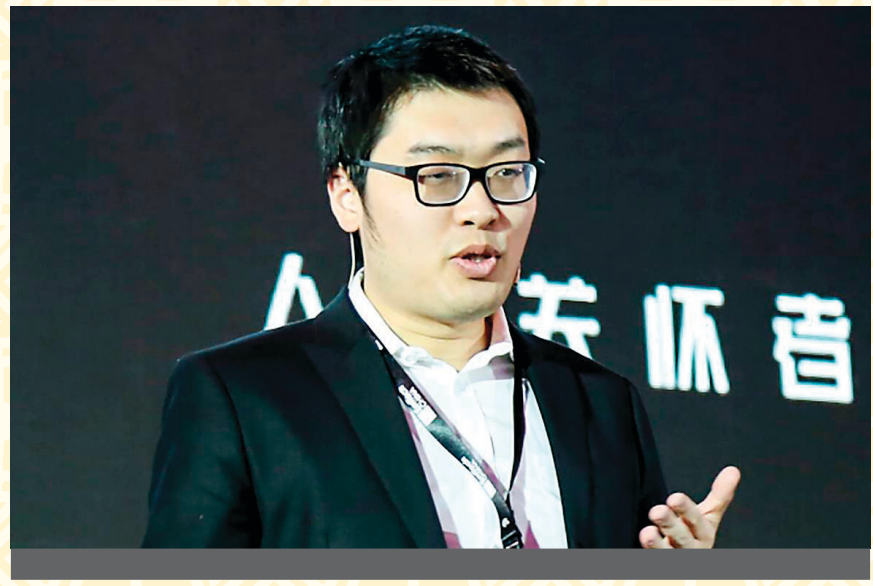

Lou Tiancheng has been called "one of the world's best hackers."

massive need for talent in China," said Jerry Yang, ${ }^{c}$ Yahoo co-founder. While plenty of jobs await computing graduates in China, some see their first or second employer as simply a steppingstone to starting their own company.

To address the technologist shortage, China aggressively recruits talent from abroad through government programs like "Thousand Talents," which through 2017 had attracted more than 7,000 top-level overseas Chinese scientists and engineers home with the promise of a 2 million yuan $(\$ 317,150)$ research grant, a 500,000 yuan "personal reward," as well as medical and housing benefits. Even major cities have entered

c https://996.ggvc.com/category/ podcast/page/2/ the recruiting act, with Shenzhen spending 500 million yuan last year under its so-called Peacock Plan to attract overseas hightech industry experts and academics.

\section{Rock Stars}

In China, tech geeks are rock stars, occupying the same limelight as teen idols. The late Stephen Hawking has a huge following in China, and Elon Musk is widely admired for his visionary work in electric cars and space travel. While some Chinese leading lights have achieved international fame, here we highlight four homegrown geek stars:

Lei Jun, CEO and cofounder of the smartphone and Internet services company Xiaomi, is a role model for young Chinese 
technologists hoping to strike it rich using their tech skills. Once referred to as the Steve Jobs of China, and derided for producing Apple copycat products, Lei has forged his own identity. Xiaomi expects to raise $\$ 6$ billion in its initial public offering (IPO). To believe Lei, money is not the motivation to build Xiaomi-he has pledged to hold profit margins on hardware down to $5 \%$, and return the surplus to users. Speaking for Xiaomi's founders, Lei has declared" "The spirit of engineering runs through our veins," and "All of us are hardcore fans of technology."

Lou Tiancheng, 32, has been called "one of the world's best hackers." He emerging as runner up at Topcoder Open in 2010 and racked up back-to-back wins at the Google Code Jam in 2008 and 2009. Lou left Baidu in December 2016 to co-found China's autonomous vehicle

d http://blog.mi.com/ en/2018/05/03/open-letter-fromour-chairman/ start-up Pony.ai, where he serves as chief technology officer. He is a role model for young Chinese techies interested in turning their coding skills into fame and fortune. While at Baidu, Lou advised $^{\text {e }}$ graduates not to think of programming as a shortcut to get into a better college or find a better job. "Learn to code for the fun of it," he said. "In the process, you will improve your reasoning and problem solving abilities."

China has 164 unicorns, which are private startups valued at more than $\$ 1 \mathrm{~B}$. The largest, with a valuation of $\$ 56 \mathrm{~B}$, is ride-hailing giant Didi Chuxing. Its president, Jean Liu, is a role model for Chinese women in tech. Born in 1978, the daughter of Liu Chuanzhi, the founder of China's computing giant Lenovo, she started the Didi Women's Network to help break what she calls the "mid-career bottleneck." Liu joined Didi in 2014 after

e http://global.baidu.com/ news-single/qa-with-top-codertiancheng-lou-baidu-autonomous-driving-team/

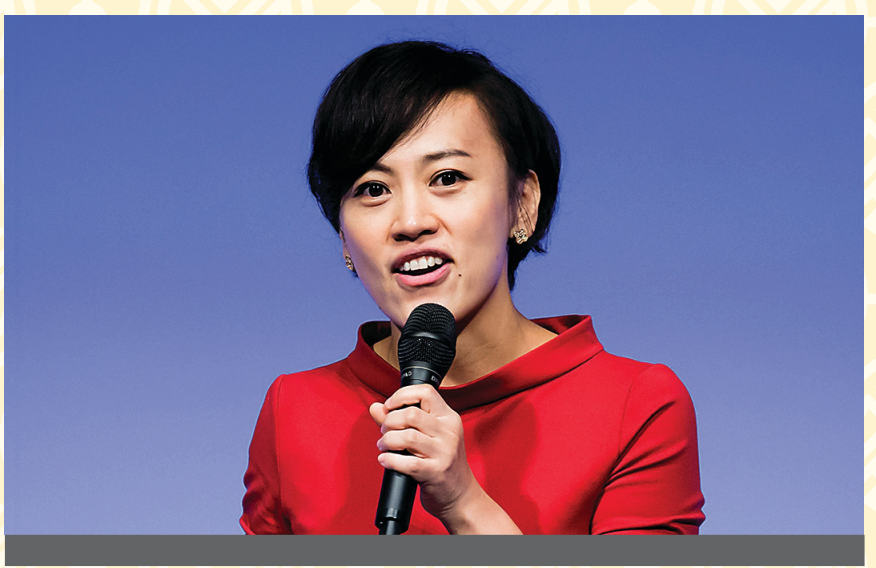

Didi president Jean Liu was named one of Time magazine's "100 Most Influential People."

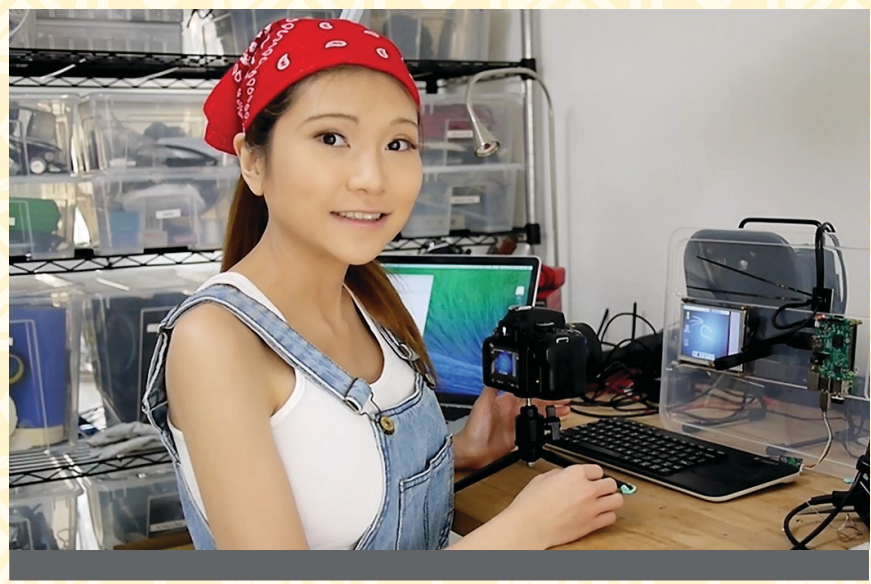

\section{Naomi Wu is on a mission to inspire women to work in the computing field.}

a career at Goldman Sachs. She was inspired to study computer science at Peking University after reading Bill Gates' The Road Ahead.

In 2017 Liu was named one of Time magazine's "100 Most Influential People," where Apple CEO Tim Cook called her a "disruptor." (Indeed, a year earlier, she convinced Apple to invest $\$ 1$ billion in Didi). After acquiring Uber's China business, she was confronted with a new crisis recently when a female passenger was raped and murdered by a Didi driver, prompting the company to tighten up security and privacy measures to protect its female riders.

While Didi, Baidu and Xiaomi are all based in Beijing, China's Silicon Valley is in Shenzhen, a metropolis of 12 million people just north of Hong Kong. Shenzhen is home to some of China's biggest tech companies, including Huawei Technologies, ZTE, drone maker DJI, and Internet giant Tencent. Shenzhen is a talent magnet, and hosts an entire hard- ware electronics ecosystem, making it an ideal base for makers-DIY techies who dabble in electronics, robotics, and 3D printing, and more.

One extraordinary maker in Shenzhen is Naomi Wu, ${ }^{\text {f }}$ an accomplished 20-something geek known as SexyCyborg to her YouTube and Twitter fans - most of whom are outside of China since both platforms are banned in China. Wu, who learned computer coding after finishing high school, is on a mission to inspire women to work in the computing field. "I'm not a rock star coder or anything," she told Newsweek in an interview in November 2017. "But I am good at cleaning up after rock star coders." Wu's DIY projects are mainly focused on wearable tech items for women.

With role models like Jun, Lou, Liu, and Wu, China's computing culture is vibrant and distinctive. c

f http://www.atimes.com/article/ meet-chinas-sexycyborg-goddess-geeks/ 